\title{
A Renaissance of Context in Design Science Research
}

\author{
Philipp zur Heiden \\ Paderborn University \\ philipp.zur.heiden@uni-paderborn.de
}

\author{
Daniel Beverungen \\ Paderborn University \\ daniel.beverungen@uni-paderborn.de
}

\begin{abstract}
Foundational theorems for sciences of the artificial highlight that the object of design is not form alone, but ensembles of form and context. However, traditional methods, frameworks, and guidelines for design science research (DSR) strongly focus on developing artifacts as forms while downplaying their contextual reference. This undue emphasis on forms leads design researchers to develop incomplete design theories. Based on drawing on the foundational literature on design as science, we advocate for a renaissance of context, leading us to propose selective adaptations of core methods and frameworks that constitute DSR. We evaluate our approach by reviewing papers that account for most of these adaptations implicitly. Further research can draw on our results to develop IT artifacts and design theories as ensembles of context and form while discussing implications for additional methods and frameworks in DSR.
\end{abstract}

\section{Introduction}

Seminal literature on design as science pointed out that design is about developing human-made artifacts (forms) that must fit the properties of the environment (context) in which they are intended to be applied [1-3]. Context has to play a crucial role in shaping an artifact's form and function, defining requirements, boundaries, and-ultimately-the utility of an artifact [1-4]. Utility is conceptualized as fit (or, more accurately: as the absence of misfit) between form and context [2]. The importance of recognizing context in design has since been inscribed into many methods that constitute the engineering sciences, including requirements engineering's purpose to identify features to be inscribed into an artifact.

Surprisingly, however, recognizing the inherent interconnection of form and context - a cornerstone of design [5-7] - seems to have been downplayed since and is now almost forgotten. The majority of methods and guidelines that design researchers use to structure their research process and report their resultshowever beneficial they may be-are limited at recognizing context's constitutional role for conducting DSR properly. Most papers treat context as a stable frame-exerting requirements that an IT artifact must fulfil to fit in - while few papers identify a need to alter a context such that it (better) fits with a (future) IT artifact. Our argument is that an IT artifact and its context interplay as a duality, and we should not overemphasize one perspective over the other when reporting DSR.

For design researchers, this means that design theories should more than before be formulated as a duality of artifact and context, specifying what properties both must satisfy to fit. From our perspective, this is the key to provide a cumulative science of design. Few DSR studies generalize their research results by abstracting ensembles of artifacts and contexts or project their results to other contexts (possible worlds) [8-10]. Instead, most papers focus on generalizing the IT artifact alone, even if many refer to the artifact's context when identifying requirements in the first stages of their design processes. Considering the importance of fit, this overemphasis of artifacts might mislead us to develop design theories incompletely.

We set out to foster a renaissance of context, reaffirming the concept in authoritative frameworks and methods that constitute the DSR paradigm. Importantly, our goal is not to define additional criteria that govern how DSR is conducted properly. Rather, our goal is to highlight that design theories become stronger if they appreciate the interplay of artifact and context more fully. Doing so could also be an important step towards fostering a cumulative research tradition in DSR, since IT artifacts could be more easily applied and extended to fit different contexts through exaptation [6].

Our contribution aims to increase both the rigor and relevance of DSR. Rigor refers to the justificatory knowledge used to design artifacts added to the knowledge base by applying DSR [1, 11, 12]. While most of the current DSR studies do not consider 
artifact and context as a duality, our results promote rigor by conceptualizing how to incorporate context as an irreducible aspect into the design and evaluation of artifacts. Relevance refers to the environment (i.e., the context) in which an artifact is applied [1, 13]. Our results promote relevance by making explicit what contexts an artifact can function in, making artifacts actionable beyond their original context.

The remainder of this paper is organized as a DSR study itself $[1,14]$, which is a common approach in IS [6]. In Section 2, we identify core problems that might follow from insufficiently considering context in a DSR paper. In Section 3, we define the objectives of re-establishing context into core artifacts of the DSR literature and, with it, propose extending the authoritative information systems research framework [1]. In Section 4, we propose updating three DSR processes with properties of context. In Section 5, we evaluate our artifacts by revisiting top-journal papers that report IT artifacts' design and evaluation. In Section 6, we discuss further implications on the core DSR literature. Section 7 concludes the paper.

\section{Problem identification and motivation}

Contrasting natural phenomena with no human control (e.g., the force of gravity), designers develop artifacts (i.e., airplanes) purposefully to serve specific functions and to imitate appearances in natural things [3]. Artifacts depict the interface between their internal environment (the artifact itself) and their surrounding (its context) [3]. In IS research, in particular, IT artifacts are intended to solve relevant problems with effectiveness or efficiency that is superior to the performance of pre-existing/rival artifacts [1, 11]. IT artifacts comprise four types: constructs, models, methods, and instantiations [11]. They are defined as objects, entities, or a combination of both, designed to benefit their users through application in a particular context $[15,16]$.

In terms of designing IT artifacts, DSR pursues a dual mission [17]: First, it contributes new theories for design and action to the knowledge base; second, it supplies IT artifacts that are actionable to solve problems in an application scenario. Therefore, DSR aims to design IT artifacts and evaluate them to build IS design theories [18]. The order of the design and evaluation steps in DSR can vary since they form a cycle for building design theories. DSR has to be relevant for practitioners, be of immediate utility [12], and needs to be performed transparently and rigorously [13], providing utility rather than truth [19].

Context, in general, is a multi-dimensional and obscure construct $[2,20]$, not clearly defined in DSR literature. One of its few definitions portrays context in organizational behavior research as "situational opportunities and constraints that affect the occurrence and meaning of organizational behavior as well as functional relationships between variables" [21, $\mathrm{p}$. 386]. Research recognizes the importance of context because contextual changes can drastically change the nature of theories [21]. Since context restricts the general applicability and universal relevance of research, it has been identified to constrain social science theories [22].

DSR is inherently dependent on considering the interplay of artifact and context for multiple reasons. First, seminal literature on design highlights the crucial position played by context, since design's ultimate goal is to achieve a fit between context and artifact (form): "When we speak of design, the real object of discussion is not form alone, but the ensemble comprising the form and its context." [2, $\mathrm{p}$. 16]. According to this definition, a context provides the problem itself, whereas artifacts are the proposed solution to the problem - the only part that designers can control directly [2]. The problem space, a part of the context, represents knowledge on how to solve a problem by constituting needs, goals, requirements, and stakeholders [4]. However, the problem space refers to the context's properties prior to the design and application of artifacts, differing from the solution space [8]. In addition, we posit that IT artifacts can exert a material agency to change their context, although this might require considerable time to change, hindering immediate DSR success. Still, designers can realize at least some control over the context by designing artifacts. We focus on investigating the interplay of context and artifact, while not referring to the problem space in this paper.

With evaluation as an essential step in design processes, Alexander [2] recommends to put the artifact into its intended context and check whether all derived requirements are fulfilled. Achieving fitness is difficult (if not impossible), though, since an obscure context can result in a virtually endless list of requirements, which forces evaluation to target the absence of misfits instead of focusing on achieving fit [2]. In line with Alexander's ideas, foundational premises of the engineering sciences shaped the foundations of DSR in IS $[1,14,18]$. Consistently, IS portrays context as differences in which studies applying DSR are executed [19], with context surrounding an IT artifact. The characteristics of context are different for each DSR project. In one of the most influential IS papers on the foundations of DSR, Hevner et al. [1] structure context as a composition of people (roles, skills, characteristics), organizations (strategies, structure, culture, processes), and technology (infrastructure, 
applications, communications architecture, development skills). Others stated similar conclusions $[23,24]$. Still, we portray the importance of context in DSR as a conjunction depending on the phase of DSR. We further differentiate between problem analysis (where the context may become a component of solution requirements), solution design (where the context may become a component of assumptions), and theorization (where the context may become a component of boundary conditions).

Second, abundant theories highlight the interplay of IT artifacts and human actions with social structures, including organizations and society as contexts with which information systems and humans interact. DSR is also subject to this claim. Meanwhile, DSR aims to solve design problems emerging from a context by building IT artifacts and evaluating the artifacts in their context [11]. However, to provide generalized statements as design theories, an IT artifact must be evaluated in different or abstracted contexts to leverage the theory's robustness [18]. On a more general level, this relates to the debate on universalism vs. particularism. Universalism demands theories to be valid outside of the context for which they were developed, to verify them in multiple contexts $[25,26]$, a principle often proclaimed as the ultimate goal of IS research [27]. Davison and Martinsons [22] oppose universalism and call for research to become more aware and dependent on context. Particularism advocates differences in the environment of theories (and, thus, their context) by considering characteristics deemed to functionally irrelevant by universalist research $[22,28]$. In a practical universalist research approach in IS, context is often neglected and should be better specified [20, 22].

While design refers to achieving fit of artifact and context, most of the current DSR papers, however, seem to overemphasize the properties of their artifacts and refer to context only in the initial steps of their design process, when defining requirements manifesting from the context. This claim is backed by a systematic literature review of DSR papers published in the IS basket journals [10], where only $17 \%$ out of 115 papers applying DSR and published in basket journals analyzed covered a description of the three context dimensions (people, organization, technology) proposed by Hevner et al. [1]. 34\% of the papers refrained from discussing relation to or implications on context that followed from their design decisions.

Considering our differentiation of context, this problem is tripartite. First, many DSR publications fail to specify the properties of context that leave their mark on their artifact and do not trace their design decisions to specific contextual characteristics (targeting problem analysis) [10]. Second, many DSR papers overemphasize describing an artifact as a form while downplaying - often even neglecting - the properties of the context for which the artifact has been designed or in which it might be used (targeting solution design) [10]. This means that IT artifacts are mostly described as the (inevitable) result of a design process rather than as a form that must fit with its context. Third, design theories are reported in terms of generalizing an IT artifact, but not in terms of generalizing the interplay of artifacts and the context in which they can be used (targeting theorization) [10].

While we think that DSR has offered important contributions, we also see that considering context more profoundly could open up new ways for reporting stronger artifacts and design theories. DSR can do more to explain the interplay on artifact and context, respecting them as two irreducible sides of a design process. Instead of theorizing the artifact only, theorizing the interplay of artifact and context could open up new ways for other researchers to apply, evaluate, extend, and replicate artifacts in other, closely related or entirely different contexts.

\section{Defining objectives of a solution}

As the term renaissance in our objective for this study indicates, we do not claim that this idea is ours alone and entirely new; instead, the call for designing ensembles of artifact and context is a foundational premise on which design has been based all along. Therefore, we propose to remind the crucial role of context and adapt methods and guidelines accordingly. DSR has produced authoritative methods and guidelines, which have proven successful and are used frequently to guide design research. Advocating a cumulative research approach in DSR, we postulate that extending the core set of methods for DSR to accommodate context and its interplay with artifacts is a more promising avenue than to design a new method from scratch.

For identifying this core set of methods, it seemed natural to focus on the papers that are most frequently applied to guide DSR studies. We applied a citation analysis on all 115 papers we identified applying DSR in the basket journals (see online appendix ${ }^{1}$ ). However, we only considered references that establish frameworks, processes, models, or guidelines for performing DSR. Some papers performed and justified their study with reference to multiple papers, whereas others did not include any reference to method papers

\footnotetext{
${ }^{1}$ https://osf.io/zm4tu/?view only=1095f57af5cc452ea45dcee48ce18be7
} 


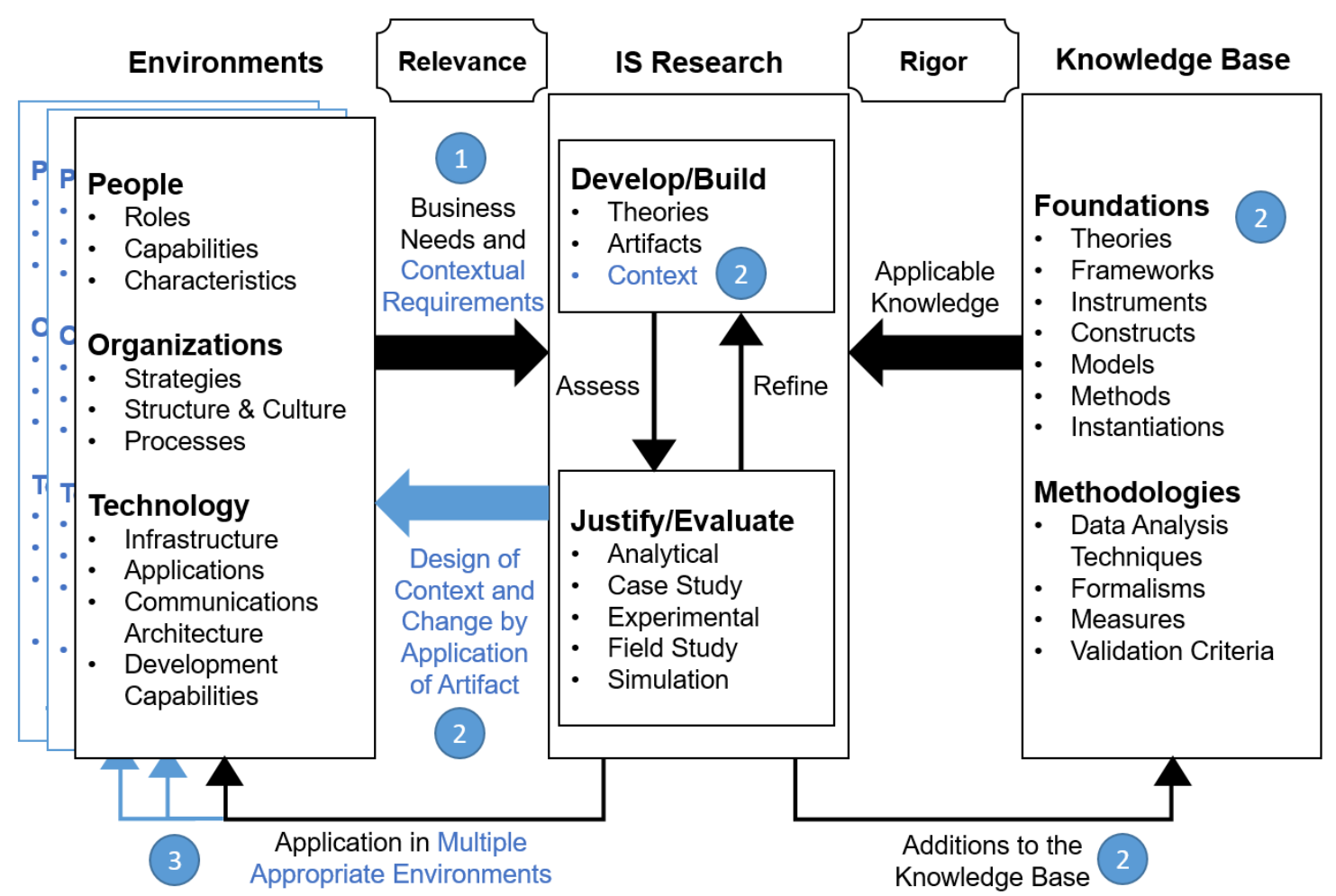

Figure 1. Modified information systems research framework, based on Hevner et al. [1]

on DSR at all. We identified 74 papers citing Hevner et al. [1], 24 referring to Peffers et al. [14], and Gregor and Hevner [6] with 16 papers as the only papers that were cited more than ten times. Our analysis corresponds to related research on DSR methods in other contexts [29]. Considering this analysis, the framework and guidelines established by Hevner et al. [1] seem best suited as a starting point for accommodating context since most other influential method papers in DSR rely on the hallmark observations stated in this work. We did not focus on Sein et al. [17] because their development of action design research substantially differs from DSR as the target of our study.

A first design step of our research is, thus, to adapt the conceptual framework by Hevner et al. [1] as an initial reference for systematizing the objectives of our solution (cf. Figure 1). As authoritative methods and guidelines for DSR itself based on this framework, we consider the frameworks, models, and guidelines developed by Peffers et al. [14], Gregor and Hevner [6], and Gregor and Jones [18] for re-introducing context.

Improving these methods, guidelines, and frameworks is a design science project itself. This meta-application of DSR to improve future DSR is in line with similar methods implemented by other researchers [6]. It is a valid approach since algorithms, models, and practices can compose the results of DSR
$[1,11]$. The additions we propose for these methods and guidelines are systematized with the framework of Hevner et al. [1] in Figure 1. The original framework is colored black, while the additions and changes we applied to it as our first design step to systematize the objectives of our solution are colored blue. The circles indicate the objectives of a solution we derived from our problem-statement: (1) consideration of context for design decisions, (2) design of the interplay of artifact and context, and (3) generalization through application in multiple contexts.

Our first objective targets the current specification and characterization of context in DSR studies. Although the environment-seen in DSR as a synonym for context - includes the identified criteria, it affects IS research in terms of business needs only. However, IS research demands recognizing the nature of context more comprehensively, to consider the implications that context has on the design of theories and artifacts [20, 22]. Thus, we include contextual implications as an effect that the environment will have on IS research. While more detailed guidance regarding context specification is provided in the framework of Hevner et al. [1], it is missing in many IS research papers that apply DSR.

Our second design objective targets the interplay of the artifact and its context. IT artifacts are inextricably linked to their context [2,3], although an artifact might be applied in different contexts. 
Nevertheless, the application of innovative artifacts as the goal of IS research [1] demands to some degree to tolerate changes in context and, to some degree, one can also change a context to appropriate an IT artifact better. For instance, a new business process (an IT artifact) might require changing the context of the process, too, establishing new roles to manage a process in context (e.g., appointing a process owner in an organization). Thus, DSR must acknowledge that beyond IT artifacts, context can — and often must- be changed as a part of the design. The degree to which context has to be (re-)designed depends on the individual DSR study and can target any or multiple of the three context dimensions. Therefore, we view the design of context as an essential part of design research in IS. Also, we recognize the effects of contextual design and application of IT artifacts on their contexts.

Our third objective refers to generalizing the results of DSR through an evaluation of the developed artifacts in their original and additional contexts. In the framework, we depict this issue by considering multiple environments (i.e., contexts) in which an artifact must be applied and evaluated to allow for proper generalization. Generalization is often seen as the ultimate goal of research because it enables universal knowledge claims [27], and as such, should be applied cautiously. Generalization in DSR revolves around the context of the IT artifacts designed [30]. Of course, the contexts in which artifacts are evaluated still have to provide similar business needs and contextual requirements. With this guideline, we propose to apply the artifact in multiple contexts that share a set of decisive properties. This guideline aims to generalize IT artifacts to classes of artifacts and context to classes of contexts - such that both artifact and context are viewed on a level of abstraction that goes beyond the properties of the current implementation and context. Thus, our results do not contrast with DSR projectability [8,9], but rather complement projectability and its application in DSR.

\section{Design Results}

\subsection{Adjusting the nominal DSR process}

Peffers et al. [14] present a nominal process for conducting DSR in IS, consisting of six activities that can be instantiated for different design projects: (1) identify the problem and motivate, (2) define objectives of a solution, (3) design and development, (4) demonstration, (5) evaluation, and (6) communication. This nominal process sequence can be re-iterated as long as the evaluation or communication results in identifying further needs to adjust the artifact. Design research, according to Peffers et al. [14], can start from any of the first four phases, depending on the purpose of the project.

We opt not to add new activities to the nominal process model since we find it to cover all relevant phases of DSR. However, we propose to extend some activities to further recognize context while designing an IT artifact. Each adjustment is underlined by at least one of our three objectives already used to adapt the information systems research framework: (obj. 1) improve context specification and consideration, (obj. 2) design ensembles including artifact and context, and (obj. 3) improve generalization through evaluating the artifact in multiple contexts.

To the first activity-identify the problem and motivate - we add a (field) description of the intended artifact's context and its dimensions, based on which requirements towards the IT artifacts and their interplay with its context can be identified (obj. 1, 2). Further, researchers ought to identify and elucidate any misfit between current artifacts and context, to state problems and motivate changes to the IT artifacts' or the context's design (obj. 3). During define objectives of a solution, objectives defined must not be limited to the IT artifact itself. Still, they must also account for the artifact's context and the interplay of the artifact and its context as an ensemble (obj. 2). Objectives of a solution ought to include a chain of causality to reason on the effects that an artifact can have on its context, and vice versa. Hypotheses could point to the desired or expected effects that artifacts can yield on their context (obj. 2). The third activitydesign and development-needs to ground design decisions in the characteristics of different context dimensions, as identified in the first activity. Any major design decisions should be documented in this way to establish a chain of evidence from the context description to the artifact's form and function (obj. 1). If appropriate, the (re-)design of an artifact's context should also be considered here, e.g., changes that must be made to the organization or other IT artifacts to apply the IT artifact (obj. 2).

We postulate that choosing a context in which to demonstrate an IT artifact appears too late to be conducted in the fourth activity-demonstrationsince any implications of context on the artifact's design could no longer change its form and function. Instead, we posit that the context in which an artifact is demonstrated needs to equal the specific context for which the artifact was designed or an instantiation of an abstract class of contexts as outlined in the artifact's design theory. In this way, the designer can evaluate the artifact in a consistent way with its structural features and the spirit of its feature set, as outlined in adaptive structuration theory [31]. 
Finally, we propose generalizing insights on IT artifacts by evaluating them in different contexts during the fifth activity-evaluation. This generalization has to be three-fold: (a) generalization of the evaluated IT artifact to a class of IT artifacts; (b) generalization of the context to a class of contexts; (c) linking the properties of context to the properties of IT artifacts, to define an ensemble (obj. 3).

\subsection{Adjusting components of a design theory}

Gregor and Jones [18] propose a way to capture, write down, and communicate design knowledge as theories for design and action [32]. These so-called design theories embody the theoretical knowledge about IT artifacts, which are either products or methods. Design theories embody principles inherent in the design of IT artifacts and are based on justificatory knowledge of both human behavior and information technology. An IS design theory is proposed to consist of eight components. The core components are (1) purpose and scope, (2) constructs, (3) principles of form and function, (4) artifact mutability, (5) testable propositions, and (6) justificatory knowledge. The additional components comprise (7) principles of implementation and (8) an expository instantiation. Design theories portray abstract, complete, and mature knowledge representations [6], making them universal theory contributions.

For purpose and scope, we recommend including a description of the context and its dimensions since context determines how an artifact must be designed to fit with its context (obj. 1, 2). We opt to adjust the constructs of the design theory by a description of the constructs that constitute context, which can target both the current context and its desired state after the design has been concluded (obj. 1, 2). Principles of form and function should include the design decisions resulting from the characteristics of different context dimensions, referring to the first component. (obj. 1). These principles of form and function should also include the effect that an IT artifact is intended to have or, more generally, might have on its context (obj. 1).

Any changes encompassed by the design theory can affect both the artifact and its context. Thus, we rename the fourth component to artifact and context mutability to render possible changes to the artifact, the context, and the interplay of both as an ensemble (obj. 2). Also, testable propositions can target the IT artifact, but also its context, and the interplay of both as ensembles, allowing for a much richer evaluation and for using a broader range of methods for theory testing (obj. 2). For justificatory knowledge, we adjust theories to target context or the interplay of artifact and context (obj. 2). Finally, for the expository instantiation, it is essential to consider a naturalistic evaluation of the artifact that explicitly refers to the artifacts' context. Artificial evaluations neglect the contextual properties of IT artifacts, leading designers to develop incomplete design knowledge. Instead, we postulate that both dimensions have to be specified to provide meaningful design knowledge (obj. 1,2).

\subsection{Adjusting the DSR publication schema}

Gregor and Hevner [6] propose seven sections for presenting a DSR study to generate rigor and relevant results: introduction, literature review, method, artifact description, evaluation, discussion, and conclusion. In contrast to the previous models, this publication scheme offers hints for writing-up DSR, guiding researchers in communicating their results for maximum impact. Nevertheless, the authors advise researchers not to follow their publication scheme blindly but to adapt it to their own needs and characteristics.

For the introduction, we recommend including a description of the context and its dimensions to specify the interplay of the artifact to be designed and its context (obj. 1, 2). Further, any misfits of current artifacts with their context should be defined, motivating the re-design of artifacts and/or their context. We find this an indispensable prerequisite to substantiate an improvement intended to demonstrate a new artifact's superiority over existing artifacts (obj. $3)$. The second phase-literature review-should include prior work ranging from theories about artifacts, omega knowledge theories about the context the artifact will be implemented into, and lambda knowledge about the interplay of artifact and context (obj. 2).

The fourth section, formerly known as artifact description, should be renamed to the artifact-context description to work for ensembles of artifact and context (obj. 1). If appropriate, authors ought to describe the (re-)design of their artifact's context, e.g., to record any changes that have to be made to the organization or to other IT artifacts to apply the IT artifact in its context (obj. 1).

For generalization purposes, we recommend researchers to include a first glimpse at the IT artifact's generalization by evaluating it in a different context during the evaluation section (obj. 3). Here, the designer must state what properties these contexts had and to what extent they differed from reasoning on the fit or misfit of their artifact with different contexts. This evaluation might identify in which contexts that artifact worked and in which its application failed, allowing the designer to formulate in which 
boundaries a design theory is expected to hold - much as in a theoretical replication that often constitutes the theory-inspired selection of cases for a multiple case study. Further, a designer may also choose to deliberately evaluate an artifact in a context for which it was not designed - as is the case in an exaptation [6] - these context's properties need to be presented vis-á-vis the properties of the context for which the artifact was built, in order to conceptualize and theorize the exaptation. In cases of designing new artifacts for new context characteristics, this classifies as an invention, abstracting the former definition from new problems to a new context. Finally, the discussion section must cover developing generalized design knowledge as theory. This generalization has to be three-fold: (a) generalize an IT artifact to a class of IT artifacts; (b) generalize a context to a class of contexts; (c) linking properties of context to properties of IT artifacts as ensembles (obj. 3).

Summarizing, we propose several additions and adjustments to three established models and frameworks for DSR in IS. As these models and frameworks are closely related and partially depend on one another, some adjustments, naturally, re-occur in all three artifacts. While we could have focused our study on re-designing just one of these artifacts, we posit strongly that - as a community of design scientists-we require all three frameworks to be consistent, to guide designers in developing, evaluating, and theorizing their ensembles. Adjusting only one of these frameworks would have led the frameworks to contradict, which could have done more harm than good for designers.

\section{Demonstration and Evaluation}

It is difficult to evaluate the extended methods based on observation, since previous DSR studies would not have applied the same methods and frameworks. In addition, applying the adjusted methods to carry out real DSR projects in different contexts was beyond this paper's scope since this paper is focused on discussing the interplay of artifact and context on an abstract level. Thus, we must leave applying the methods and frameworks in real DSR projects to future research.

While we intentionally refrained from developing and designing entirely new models and frameworks, we performed a conceptual evaluation similar to those presented in the original papers. We reviewed recent applications of these methods in IS basket journals to elucidate how the authors, maybe implicitly, adhered to the adjustments we propose here. This approach is a naturalistic ex-ante evaluation [33]. We highlight that the positive approach we take here is very similar to the approaches that were taken in many other authoritative method design studies [1, 6, 14, 17].

For consistency with the updated methods and guidelines we propose, the evaluation had to be performed in multiple contexts for the IT artifact due to DSR. Thus, we had to consider different streams of IS research, referring to different technologies, journals, and people. In doing so, we consider different instantiations of context characteristics [1]. For our evaluation, we select three papers based on the following inclusion criteria: publication in leading journal of IS discipline, different journal and different authors, adherence to DSR model or framework, and favoring papers recently published.

Case 1: Designing In-Home Monitoring Systems for Diabetes Patients. Chatterjee et al. [34] design and evaluate IT artifacts for supporting diabetes patients in their homes by capturing daily activity and motivating them to extend their physical activity. The authors apply persuasive sensing with the Internet of Things (IoT) and mobile phones. They develop analytical models to predict the blood glucose levels of patients for the following day. This paper applies the methodology proposed by Peffers et al. [14]. For the first activity-identify the problem and motivateChatterjee et al. [34] describe all three context dimensions and their interplay with the IT artifacts they develop. To identify misfits between context and current IT artifacts, the authors discuss aspects of how their solution might improve beyond rival IT artifacts.

Chatterjee et al. [34] define their objective to improve the quality of their patients' lives by enabling them to adhere to improved self-management. They strive to improve the people-specific context based on applying their artifacts. The authors describe the problem they solve from the healthcare problem's domain perspective, not as a technology problem. They focus on patients' well-being and, therefore, use simple technologies that do not go beyond the technological state-of-the-art. Their goal is to improve the physical activity and health status of their patients after using the artifact. Thus, they implicitly opted for the consideration of context advocated here.

For the third activity — design and developmentthe authors systematically grounded their design decisions on contextual characteristics, e.g., using advantages of the IoT for a system that works in their subjects' homes. Further, they design the technological context to provide Internet and installing sensors in specific furniture. The authors evaluate their artifact in two different contexts, but these seem very similar and only differ regarding people-specific characteristics. Still, the authors generalize both the artifact and its context as "sensor-based in-home monitoring systems to assist a particular health condition" [34]. 
Case 2: Designing Shopping Assistance Artifacts in Customers' Mobile Phones. Venkatesh et al. [35] design and evaluate six shopping assistance artifacts manipulated by hardware and content design. For hardware, they either use barcode scanners or radiofrequency identification (RFID), whereas, for content design, they use product information, product reviews, and a combination of both. All artifacts are intended to be used on customers' mobile phones.

The authors mostly align with the adjustments we developed for the components of an IS design theory by Gregor and Jones [18]. They consider people as retail shoppers, technology as smartphones, and organizations as retailers and (commercial) customers. As for the constructs included in the IS design theory, the authors identify technology adoption, security beliefs, and shopping outcome. For principles of form and function, the authors align with our adjustments, e.g., by justifying why they choose RFID and barcode scanners after analyzing context characteristics, especially concerning the dimensions of people and organizations. As effects that artifacts might exert on context, however, the authors do not describe changes of context to be expected from using or deploying their artifact. For "artifact and context mutability," the authors refer to adaptions of the artifact and its flexible design, making it adjustable to various contexts.

As for testable propositions, the authors target the influence of their artifact on its context. They do this by proposing technology adoption outcomes, security beliefs, and shopping outcomes as consequences of using their artifact. All of these propositions seem highly related to the interplay of the artifact and its context. Justificatory knowledge - the sixth component-also aligns with our adjustments since the authors, again, refer to context-related knowledge, e.g., technology adaption and consumer behavior.

The authors evaluate their artifacts with an (artificial) experiment and do not refer to contextual properties they deem relevant or present in a real-life context in which their artifact will be used. Nevertheless, the experiments take place in settings resembling retail stores' properties as far as possible, therefore, resembling a natural context.

Case 3: Designing Mobile Peer Groups for Career Counseling. Klier et al. [36] design and develop a novel approach to career counseling by providing peer groups with a mobile IT artifact to support one another independently of time and place to improve career opportunities and to counteract youth unemployment: mobile peer groups. The paper not only aligns with the publication scheme in general but also follows our adjustments for proper context consideration in DSR. In their introductory sections, the authors describe the contextual characteristics, including ICT and mobile phones (technological context), career counseling with face-to-face relationships between counselors and clients (organizational context), and young people in danger of youth unemployment (people-specific context). Further, Klier et al. [36] include theory concerning the interplay of artifact and context. Justificatory knowledge includes youth unemployment, peer group mechanisms in online and offline scenarios, and potential effects of mobile IT artifacts on context. They only miss out on identifying misfits of rival artifacts and their context in the introduction, perhaps due to a lack of these artifacts.

Klier et al. [36] excel at their evaluation with a field experiment covering different cities, different counselors, and youths of different school systems. Thus, the artifact is evaluated in different natural contexts. Further, the authors demonstrate the benefits of the class of IT artifacts they design and extend their research to other domains, e.g., healthcare, education, and parenting.

\section{Discussion}

We identified and motivated problems related to DSR papers' insufficient consideration of context. We developed objectives of a solution to foster a renaissance of context in authoritative methods and guidelines of the DSR field, reviving core observations of design that were established in the early days of sciences of the artificial. We aim at considering the interplay of IT artifacts and contexts, beyond developing artifacts and IS design theories that abstract from artifacts only.

During the demonstration and evaluation of three different cases of DSR in IS, we see publications that apply some of the adjustments we designed for improving current models and frameworks for DSR publication in IS. The evaluation, thus, suggests that the models and guidelines that constitute DSR might have to be extended, too, to fit the standards of recent top-journal papers published in DSR. Beyond the papers we used as illustrative and best practice examples, however, we found that many other DSR papers published in leading IS journals do not pay particular (if any) attention to their artifacts' contexts.

While our study is about re-introducing context into methods and guidelines of DSR in IS, we had to consider DSR's meta-context to satisfy our standards of considering context in design. For identifying the problem and motivating our research, we identified the IS discipline as our context with its different subdomains and research streams, portrayed by different authors using different design science methods. Defining this meta-context consistently is problematic 
since the context itself is obscure [20] and cannot always be abstracted. We discussed why current artifacts-methods and guidelines for DSR that constitute the core of this field-insufficiently prescribe ways to consider the context in DSR. However, we did not change the context of our study, as we believe that changes on a meta-theoretical level cannot be made within a single publication. We hope to encourage other researchers, reviewers, and editors to focus more on considering the context in their DSR studies to further improve DSR's rigor and relevance.

Concerning developing generalized theory, the methods and guidelines extended here target DSR in IS. Nevertheless, our results can also be applied in neighboring disciplines, e.g., engineering, computer science, and marketing. As we consider IS as our primary research field, we evaluated the artifact by reviewing applications of DSR in different contexts inside the IS discipline. We covered IS top-journals to identify different publications, authors, artifact types, and contexts. Future research might provide successful applications of the adaptations we proposed.

IS, as an interdisciplinary research discipline, investigates both technological and social phenomena [37]. Thus, our research has to adhere to technical and social research. Social sciences distinguish a nomothetic knowledge scope - abstracting insight to general and universal knowledge-from an idiographic knowledge scope-focusing on situated artifacts and particular instantiations [38]. Results of DSR can differ in terms of the knowledge scope they deliver and might also cover multiple knowledge scopes in a single study [38]. In general, instantiations without an abstraction contain an idiographic knowledge scope, whereas high-level design theories tend to display a nomothetic knowledge scope [6]. However, DSR should contain a combination of both kinds of knowledge scopes [38]. As our adjusted methods and guidelines target a proper generalization of context and artifact, we aim to enable future DSR research to encompass both a nomothetic and an idiographic knowledge scope.

To categorize the contributions of DSR, Gregor and Hevner [6] present a knowledge contribution framework. Their $2 \times 2$ matrix systematizes DSR contributions in terms of their solution maturity and their application domain maturity. We consider the contribution offered here to display high application domain maturity since aligning artifact and context has been characterized as a hallmark property of design from the early days of the sciences of the artificial. The solution maturity of considering context is low since existing methods and guidelines insufficiently address the interplay of artifact and context in DSR. Thus, we consider our IT artifacts as improvements.

\section{Conclusion}

Our paper contributes to theory by underlining the importance of considering ensembles of artifacts and context in design and DSR. Our guidelines increase rigor and relevance in DSR since they serve to make explicit subject to which contexts and artifacts will function and IS design theories will apply. The proposed additions provide hands-on guidelines for researchers to consider more properly in DSR projects.

Limitations comprise not considering any direct applications of the proposed additions to methods and guidelines. Investigating the consensus of recent DSR publications with our methods and guidelines seemed like an adequate starting point to check the applicability of these propositions, though. After all, neither of the methods we extended was applied and evaluated based on applying them to a real design science project reported in the original paper. From a methodological point of view, our naturalistic ex-ante evaluation is subject to the risk of identifying false positives [33]. An entirely different approach might have been to review other DSR papers and identify to what extent they failed to consider context properly, but we felt that using new criteria to highlight deficiencies in papers would have been unfair. Further research is required to validate, extend, and theorize the adjusted methods and guidelines by applying them to the contexts of different design projects. Our common goal should be to promote DSR as a research paradigm, fostering DSR to be published more frequently in the top journals of the IS discipline.

Acknowledgements: This paper was developed in the research project FLEMING, which is funded by the German Federal Ministry for Economic Affairs and Energy (BMWi), promotion sign 03E16012F.

\section{References}

[1] A. R. Hevner, S. T. March, J. Park, and S. Ram, "Design Science in Information Systems Research," MIS Quarterly, vol. 28, no. 1, pp. 75-105, 2004.

[2] C. Alexander, Notes on the Synthesis of Form. Cambridge, Mass.: Harvard Univ. Press, 1964.

[3] H. A. Simon, The Sciences of the Artificial, 3rd ed. Cambridge, Mass: MIT Press, 1996.

[4] A. Maedche, S. Gregor, S. Morana, and J. Feine, "Conceptualization of the Problem Space in Design Science Research,” DESRIST 2019 proceedings.

[5] R. Winter, "Design Science Research in Europe," EJIS, vol. 17, no. 5, pp. 470-475, 2008.

[6] S. Gregor and A. R. Hevner, "Positioning and Presenting Design Science Research for Maximum Impact," MIS Quarterly, vol. 37, no. 2, pp. 337-355, 2013. 
[7] S. Purao, "Truth or Dare," Journal of Database Management, vol. 24, no. 3, pp. 51-66, 2013.

[8] J. Vom Brocke, R. Winter, A. Hevner, and A. Maedche, "Special Issue Editorial-Accumulation and Evolution of Design Knowledge in Design Science Research: A Journey Through Time and Space," JAIS, vol. 21, no. 3, pp. 520-544, 2020.

[9] R. Baskerville and J. Pries-Heje, "Projectability in Design Science Research," JITTA, vol. 20, no. 1, 2019.

[10] P. zur Heiden, "Considering Context in Design Science Research: A Systematic Literature Review," in LNCS, vol. 12388, DESRIST 2020 proceedings, S. Hofmann, Ed.: Springer, 2020, pp. 223-234.

[11] S. T. March and G. F. Smith, "Design and Natural Science Research on Information Technology," Decision Support Systems, vol. 15, no. 4, pp. 251266, 1995.

[12] B. Kuechler and V. Vaishnavi, "Promoting Relevance in IS Research: An Informing System for Design Science Research," Informing science: The international journal of an emerging transdiscipline, vol. 14, no. 1, pp. 125-138, 2011.

[13] J. F. Nunamaker, M. Chen, and T. D. M. Purdin, "Systems Development in Information Systems Research,” JMIS, vol. 6, no. 4, pp. 89-106, 1990.

[14] K. Peffers, T. Tuunanen, M. A. Rothenberger, and S. Chatterjee, "A Design Science Research Methodology for Information Systems Research," JMIS, vol. 24, no. 3, pp. 45-77, 2007.

[15] W. J. Orlikowski and S. Iacono, "Research Commentary: Desperately Seeking the IT in IT Research - A Call to Theorizing the IT Artifact," ISR, vol. 12, pp. 121-134, 2001.

[16] P. Zhang, M. J. Scialdone, and M.-C. Ku, "IT Artifacts and the State of IS Research," in Proceedings of the ICIS 2011, 2011.

[17] M. K. Sein, O. Henfridsson, S. Purao, M. Rossi, and R. Lindgren, "Action Design Research," MIS Quarterly, vol. 35, no. 1, p. 37, 2011.

[18] S. Gregor and D. Jones, "The Anatomy of a Design Theory," JAIS, vol. 8, no. 5, pp. 312-335, 2007.

[19] J. Iivari, "Distinguishing and Contrasting Two Strategies for Design Science Research," EJIS, vol. 24, no. 1, pp. 107-115, 2015.

[20] S. Sarker, "Building on Davison and Martinsons' Concerns: A Call for Balance between Contextual Specificity and Generality in IS Research," JIT, vol. 31, no. 3, pp. 250-253, 2016.

[21] G. Johns, "The Essential Impact of Context on Organizational Behavior," Academy of Management Review, vol. 31, no. 2, pp. 386-408, 2006.

[22] R. M. Davison and M. G. Martinsons, "Context is King! Considering Particularism in Research Design and Reporting," JIT, vol. 31, no. 3, pp. 241-249, 2016.

[23] A. Alturki, G. G. Gable, and W. Bandara, "A Design Science Research Roadmap," DESRIST 2011 proceedings, vol. 32, no. 4, pp. 107-123, 2011.

[24] A. Dresch, D. P. Lacerda, and J. A. Antunes Júnior, Design Science Research: A Method for Science and
Technology Advancement. Cham and s.l.: Springer Intern. Publishing, 2015.

[25] A. S. Lee and R. Baskerville, "Conceptualizing Generalizability: New Contributions and a Reply," MIS Quarterly, vol. 36, no. 3, p. 749, 2012.

[26] P. B. Seddon and R. Scheepers, "Generalization in IS Research: a Critique of the Conflicting Positions of Lee \& Baskerville and Tsang \& Williams," in Formulating Research Methods for Information Systems: Volume 1, L. Willcocks, C. Sauer, and M. C. Lacity, Eds., New York, NY: Palgrave Macmillan, 2015, pp. 179-209.

[27] Z. Cheng, A. Dimoka, and P. A. Pavlou, "Context May Be King, but Generalizability is the Emperor!," JIT, vol. 31, no. 3, pp. 257-264, 2016.

[28] J. S. Long and M. F. Fox, "Scientific Careers: Universalism and Particularism," Annual Review of Sociology, vol. 21, no. 1, pp. 45-71, 1995.

[29] A. Cater-Steel, M. Toleman, and M. M. Rajaeian, "Design Science Research in Doctoral Projects: An Analysis of Australian Theses," JAIS, vol. 20, no. 12, 2019.

[30] J. E. van Aken, "Management Research Based on the Paradigm of the Design Sciences: The Quest for FieldTested and Grounded Technological Rules," Journal of Management Studies, vol. 41, no. 2, pp. 219-246, 2004.

[31] G. DeSanctis and M. Poole, "Capturing the Complexity in Advanced Technology Use: Adaptive Structuration Theory," Organization Science, vol. 5, no. 2, pp. 121-147, 1994.

[32] Gregor, "The Nature of Theory in Information Systems," MIS Quarterly, vol. 30, no. 3, pp. 611-642, 2006.

[33] J. Venable, J. Pries-Heje, and R. Baskerville, "A Comprehensive Framework for Evaluation in Design Science Research," in DESRIST 2012 proceedings: Springer, 2012, pp. 423-438.

[34] S. Chatterjee, J. Byun, K. Dutta, R. Pedersen, A. Pottathil, and H. Xie, "Designing an Internet-ofThings (IoT) and Sensor-based In-Home Monitoring System for Assisting Diabetes Patients: Iterative Learning from two Case Studies," EJIS, vol. 27, pp. $1-16,2018$

[35] V. Venkatesh, J. A. Aloysius, and S. Burton, "Design and Evaluation of Auto-ID Enabled Shopping Assistance Artifacts in Customers' Mobile Phones: Two Retail Store Laboratory Experiments," MIS Quarterly, vol. 41, no. 1, pp. 83-113, 2017.

[36] J. Klier, M. Klier, L. Thiel, and R. Agarwal, "Power of Mobile Peer Groups: A Design-Oriented Approach to Address Youth Unemployment," Journal of MIS, vol. 36, no. 1, pp. 158-193, 2019.

[37] M. Tarafdar and R. Davison, "Research in Information Systems: Intra-Disciplinary and InterDisciplinary Approaches," JAIS, vol. 19, no. 06, pp. 523-551, 2018.

[38] R. Baskerville, M. Kaul, and V. C. Storey, "Genres of Inquiry in Design-Science Research: Justification and Evaluation of Knowledge Production," MIS Quarterly, vol. 39, no. 3, pp. 541-564, 2015. 